\title{
ON THE POLYNOMIAL LIMIT CYCLES OF POLYNOMIAL DIFFERENTIAL EQUATIONS
}

\author{
JAUME GINÉ ${ }^{1}$, MAITE GRAU ${ }^{1}$ AND JAUME LLIBRE ${ }^{2}$
}

\begin{abstract}
In this paper we deal with ordinary differential equations of the form $d y / d x=P(x, y)$ where $P(x, y)$ is a real polynomial in the variables $x$ and $y$, of degree $n$ in the variable $y$. If $y=\varphi(x)$ is a solution of this equation defined for $x \in[0,1]$ and which satisfies that $\varphi(0)=\varphi(1)$, we say that it is a periodic orbit. A limit cycle is an isolated periodic orbit in the set of all periodic orbits. If $\varphi(x)$ is a polynomial, then $\varphi(x)$ is called a polynomial solution.

We study the maximum number and the multiplicity of the polynomial limit cycles of $d y / d x=P(x, y)$ with respect to $n$. We prove that this differential equation has at most $n$ polynomial limit cycles and that this bound is sharp. If $n=1$ (linear equation), or $n=2$ (Riccati equation), we prove that the differential equation $d y / d x=P(x, y)$ has at most $n$ polynomial limit cycles counted with their multiplicities. For $n=3$ (Abel equation) we show that at most three polynomial limit cycles can exist whereas the multiplicity of a polynomial limit cycle can be unbounded.
\end{abstract}

\section{IntRoduction AND STATEMENT OF the MAin RESUlts}

We consider ordinary differential equations of the form

$$
\frac{d y}{d x}=A_{0}(x)+A_{1}(x) y+A_{2}(x) y^{2}+\ldots+A_{n}(x) y^{n}
$$

where $x$ and $y$ are real variables, $A_{i}(x)$ are polynomials in $\mathbb{R}[x]$ for $i=$ $0,1,2, \ldots, n$, and $n$ is an integer number with $n \geq 1$. We assume that $A_{n}(x)$ is not identically zero. We shall denote the derivative of $y$ with respect $x$ by $d y / d x$ or $y^{\prime}$.

First we are interested in the polynomial solutions $y=p(x)$ of the differential equation (1), i.e. in the solutions $y=p(x)$ of (1) where $p(x)$ is a polynomial.

A periodic solution of (1) is a solution $y=\varphi(x)$ of (1) such that $\varphi$ : $[0,1] \rightarrow \mathbb{R}$ is $C^{1}$ and $\varphi(0)=\varphi(1)$. We say that such a periodic solution has period 1 . In order to define a periodic solution, we can choose any period, but by an affine change of the independent variable $x$, there is no loss of generality in searching for the periodic solutions of period 1.

2000 Mathematics Subject Classification. 34C05, 34C25, 58F21.

Key words and phrases. limit cycles, polynomial differential equations, multiplicity, bifurcation. 
Let $\mathcal{E}$ be the space of the $C^{1}$ functions from $[0,1]$ to $\mathbb{R}$ satisfying $\varphi(0)=$ $\varphi(1)$ with the topology of the supremum. We say that a periodic solution $y=\varphi(x)$ of (1) is a limit cycle of (1) if in $\mathcal{E}$ there exists a neighborhood of $\varphi$ without any other periodic solution of (1); i.e. $y=\varphi(x)$ is isolated in the set of all periodic solutions of (1).

We denote by $\psi\left(x ; y_{0}\right)$ the solution of the differential equation $(1)$ such that $\psi\left(0 ; y_{0}\right)=y_{0}$. The translation operator associated to equation (1) is the function $\Psi: \mathbb{R} \rightarrow \mathbb{R}$ such that $\Psi\left(y_{0}\right)=\psi\left(1 ; y_{0}\right)-y_{0}$. We see that $\Psi\left(y_{0}\right)$ is defined if and only if the solution $\psi\left(x ; y_{0}\right)$ exists for $x \in[0,1]$. Clearly from this definition we have that $\Psi\left(y_{0}\right)=0$ if and only if equation (1) has a periodic solution starting at $y_{0}$. Therefore there is a one-toone correspondence between the zeros of the translation operator and the periodic orbits of system (1). Following to Lloyd [8,9] the multiplicity of a limit cycle of (1) associated to the isolated zero $y_{0}$ of the translation operator is the multiplicity of $y_{0}$ as a zero of $\Psi\left(y_{0}\right)$. A periodic solution of multiplicity 1 will be said to be simple, or hyperbolic.

We are mainly interested in the limit cycles, polynomial or not, that a differential equation (1) can exhibit and in knowing the answers to the following questions: When the limit cycles of the differential equation (1) are polynomial? What are the maximum number of polynomial limit cycles that the differential equation (1) can have with respect to $n$ ? Which can be the multiplicities of such limit cycles?

The computation of exact solutions (such as polynomial and rational solutions) of a nonlinear differential equation is of great interest to understand the whole set of solutions and its dynamical properties. In 1936 Rainville [10] determines all the Riccati differential equations of the form $y^{\prime}=A_{0}(x)+A_{1}(x) y+y^{2}$, with $A_{0}$ and $A_{1}$ polynomials, which have polynomial solutions; and provides an algebraic method to compute those polynomial solutions.

Campbell and Golomb [5] in 1954 provide an algorithm for finding all the polynomial solutions of the differential equation $B(x) y^{\prime}=A_{0}(x)+A_{1}(x) y+$ $A_{2}(x) y^{2}$, where $B, A_{0}, A_{1}$ and $A_{2}$ are polynomials in $x$. More recently Behloul and Cheng [1] gave another algorithm to look for all the rational solutions of equations of the form

$$
B(x) \frac{d y}{d x}=A_{0}(x)+A_{1}(x) y+A_{2}(x) y^{2}+\ldots+A_{n}(x) y^{n},
$$

where $B$ and $A_{i}$ for $i=0,1,2, \ldots, n$ are polynomials in $x$. They first simplify this search to find the polynomial solutions of an easier equation. They show that the polynomial solutions of this easier equation have degree lower than a uniform bound and then, by imposing a polynomial of feasible degree, they provide an algorithm for computing all the polynomial solutions.

The differential equations (1) for $n=3$ are the Abel differential equations. These equations have been studied intensively, either looking for their 
centers, i.e. for a continuum of periodic orbits, see the works of Briskin, Françoise and Yomdin [2, 3, 4]; or studying their periodic orbits, see for instance [6] or [7].

The following result establishes the maximum number of polynomial solutions and periodic polynomial solutions that a differential equation (1) can have.

Theorem 1. The following statements hold.

(a) Any differential equation (1) has at most n polynomial solutions and there are examples of equations (1) with exactly $n$ polynomial solutions.

(b) The difference between two polynomial solutions of equation (1) is a constant.

(c) If (1) has a polynomial periodic solution, then all polynomial solutions are periodic.

(d) Any equation of the form (1) has at most $n$ polynomial periodic solutions and there are examples of equations of the form (1) with exactly $n$ polynomial periodic solutions.

From Theorem 1 it follows immediately the next result.

Corollary 2. Any differential equation (1) has at most $n$ polynomial limit cycles and there are examples of equations (1) with exactly $n$ of such cycles.

We are also interested in the multiplicity of the polynomial limit cycles of the differential equations (1).

The number and multiplicity of limit cycles of the differential equations (1) with $n=1$ (linear equation) and $n=2$ (Riccati equation) were studied by Lloyd [8] and Lins Neto [7]. The next result shows that the upper bounds described in these previous works are also realizable only taking into account polynomial limit cycles.

Theorem 3. The following statement hold.

(a) Assume that a differential equation (1) with $n=1$ (linear equation) has a periodic solution. If $\alpha=\int_{0}^{1} A_{1}(x) d x \neq 0$, then this periodic solution is the unique limit cycle of equation (1) and it is hyperbolic. If $\alpha=0$ the equation has a continuum of periodic solutions.

(b) Assume that a differential equation (1) with $n=2$ (Riccati equation) has a periodic solution $y=p(x)$. Define $\beta=\mathcal{D}_{1}(1)$ and $\gamma=\int_{0}^{1} e^{\mathcal{D}_{1}(s)} A_{2}(s) d s$, where $\mathcal{D}_{1}(s)=\int_{0}^{s}\left(A_{1}(\sigma)+2 p(\sigma) A_{2}(\sigma)\right) d \sigma$. If $\beta^{2}+\gamma^{2} \neq 0$, then the equation has at most either two limit cycles (both hyperbolic), or one limit cycle of multiplicity 2. In these two last cases one of the limit cycles is the periodic orbit $y=p(x)$. If $\beta=\gamma=0$, then the equation has a continuum of periodic solutions. 
While the number and multiplicity of limit cycles for the differential equations (1) with $n=1$ and $n=2$ is always bounded, this is not the case for $n \geq 3$. If we only take into account polynomial limit cycles, we have that their number is bounded as Corollary 2 states, but not their multiplicity as the next result shows. This result will be proved by using ideas from Lins Neto [7], who proved the existence of an Abel differential equation with as many limit cycles as desired. In the work [6] some sufficient conditions on the coefficients of the Abel differential equation are given in order to bound the number of its limit cycles.

Theorem 4. Given any integer number $m$ with $m \geq 2$, there exist polynomials $A_{2}(x)$ and $A_{3}(x)$ such that $y=0$ is a polynomial limit cycle of multiplicity $m$ of the Abel differential equation

$$
\frac{d y}{d x}=A_{2}(x) y^{2}+A_{3}(x) y^{3} .
$$

In the proof of this theorem, given in section 3, we give an example of an Abel differential equation with $A_{2}(x)$ a polynomial of degree 1 and $A_{3}(x)$ a polynomial of degree $2(m-3)$ having $y=0$ as a polynomial limit cycle of multiplicity $m$.

In general it is not possible to integrate an Abel differential equation if only one particular solution is known, whereas for a Riccati equation is always possible as it is well-known (and as in particular we will show in the proof of Theorem 3). Actually even the knowledge of a countable number of explicit solutions of an Abel differential equation does not seem to imply its explicit integration. This fact together with the iterated integrals that appear in the determination of the multiplicity of a limit cycle, see Lemma 5 , avoid that in general an Abel differential equation has a uniform bound for the multiplicity of its polynomial limit cycles.

The rest of the paper is structured as follows. In section 2 we prove Theorem 1, and in section 3 we provide the proofs of Theorems 3 and 4 .

\section{Polynomial limit cycles}

In this section we mainly prove Theorem 1.

Proof of Theorem 1. Let $y=p(x)$ be a polynomial solution of (1). We consider the following affine change in the dependent variable $y \mapsto z$ with $z=y-p(x)$, which transforms equation (1) into

$$
\frac{d z}{d x}=\tilde{A}_{1}(x) z+\tilde{A}_{2}(x) z^{2}+\tilde{A}_{n-1}(x) z^{n-1}+A_{n}(x) z^{n},
$$

where each $\tilde{A}_{i}$ is a polynomial depending on the $A_{j}(x)$ for $j=i, i+1, \ldots, n$ and on some powers of $p(x)$, for $i=1,2, \ldots, n$.

We observe that any polynomial solution of (1) gives rise to a polynomial solution of (2), and vice versa any polynomial solution of (2) gives rise to a polynomial solution of (1). In particular the constant solution $z=0$ 
corresponds to $y=p(x)$ for $(1)$. Let $z=q(x)$ be another polynomial solution of (2) different from $z=0$, that is

$$
q^{\prime}(x)=\tilde{A}_{1}(x) q(x)+\tilde{A}_{2}(x) q(x)^{2}+\tilde{A}_{n-1}(x) q(x)^{n-1}+A_{n}(x) q(x)^{n} .
$$

Then, since $q(x)$ divides the right-hand side of the latter expression, we have that it also divides the left-hand side, that is, $q(x) \mid q^{\prime}(x)$. This fact implies that $q(x)$ is a constant and we denote it by $q(x) \equiv \zeta \in \mathbb{R}$. We have that any polynomial solution of (2) needs to be a constant.

We deduce that the polynomial in $z$ given by $\tilde{A}_{1}(x) z+\tilde{A}_{2}(x) z^{2}+$ $\tilde{A}_{n-1}(x) z^{n-1}+A_{n}(x) z^{n}$ is divisible by $(z-\zeta)$ and, since its degree in $z$ is $n$ and $z=0$ is already a root, we have that it has, at most, $n$ different constant roots. Going back to equation (1), we deduce that equation (1) has at most $n$ polynomial solutions and that the difference between two polynomial solutions of (1) is a constant.

We remark that the differential equation

$$
\frac{d y}{d x}=y(y-1)(y-2) \ldots(y-(n-1)),
$$

is of the form (1) and has exactly $n$ polynomial solutions.

We have proved statements (a) and (b). We also remark that the latter differential equation also provides the proof of statement (d) as any constant solution is a periodic orbit. Finally note that statement (c) is a direct consequence of statement (b).

From the previous proof, we observe that if an equation (1) of degree $n$ in $y$ has $k$, with $k \leq n$, polynomial solutions, then it can be written (after a polynomial transformation) into the form

$$
\frac{d y}{d x}=r(x, y)\left(y-c_{1}\right)\left(y-c_{2}\right) \ldots\left(y-c_{k}\right)
$$

where $c_{i} \in \mathbb{R}, i=1,2, \ldots, k$, and $r(x, y)$ is a real polynomial of degree $n-k$ in $y$.

\section{Limit CYClES AND THEIR MULTIPLiCITIES}

We are going to study the multiplicity of the limit cycles of the differential equations (1) using ideas from [9].

Lemma 5. Consider the differential equation $d y / d x=\mathcal{F}(x, y)$ where $\mathcal{F}$ is a function of class $\mathcal{C}^{5}$ on $\mathbb{R}^{2}$. Assume that $y=\varphi(x)$ is a periodic orbit of this equation. Then it is

(i) a hyperbolic limit cycle if and only if $\mathcal{D}_{1}(1) \neq 0$;

(ii) a limit cycle of multiplicity 2 if and only if, $\mathcal{D}_{1}(1)=0$ and $\mathcal{D}_{2}(1) \neq 0$;

(iii) a limit cycle of multiplicity 3 if and only if $\mathcal{D}_{1}(1)=\mathcal{D}_{2}(1)=0$ and $\mathcal{D}_{3}(1) \neq 0$

(iv) a limit cycle of multiplicity 4 if and only if $\mathcal{D}_{1}(1)=\mathcal{D}_{2}(1)=\mathcal{D}_{3}(1)=$ 0 and $\mathcal{D}_{4}(1) \neq 0$; 
(v) a limit cycle of multiplicity $\geq 5$ or it belongs to a continuum of periodic orbits if $\mathcal{D}_{1}(1)=\mathcal{D}_{2}(1)=\mathcal{D}_{3}(1)=\mathcal{D}_{4}(1)=0$.

where

$$
\begin{aligned}
& \mathcal{D}_{1}(x)=\int_{0}^{x} \frac{\partial \mathcal{F}}{\partial y}(\sigma, \varphi(\sigma)) d \sigma \\
& \mathcal{D}_{2}(x)=\int_{0}^{x} e^{\mathcal{D}_{1}(s)} \frac{\partial^{2} \mathcal{F}}{\partial y^{2}}(s, \varphi(s)) d s \\
& \mathcal{D}_{3}(x)=\int_{0}^{x} e^{2 \mathcal{D}_{1}(s)} \frac{\partial^{3} \mathcal{F}}{\partial y^{3}}(s, \varphi(s)) d s \\
& \mathcal{D}_{4}(x)=\int_{0}^{x} e^{3 \mathcal{D}_{1}(s)} \frac{\partial^{4} \mathcal{F}}{\partial y^{4}}(s, \varphi(s)) d s+ \\
& +2 \int_{0}^{x} e^{2 \mathcal{D}_{1}(s)} \frac{\partial^{3} \mathcal{F}}{\partial y^{3}}(s, \varphi(s))\left(\int_{0}^{s} e^{\mathcal{D}_{1}(\xi)} \frac{\partial^{2} \mathcal{F}}{\partial y^{2}}(\xi, \varphi(\xi)) d \xi\right) d s .
\end{aligned}
$$

Proof. We consider the differential equation $d y / d x=\mathcal{F}(x, y)$ and we assume that $y=\varphi(x)$ is a periodic solution of this equation. We take the following change of the dependent variable $z=y-\varphi(x)$ and we obtain the following differential equation:

$$
\frac{d z}{d x}=\mathcal{F}(x, z+\varphi(x))-\mathcal{F}(x, \varphi(x)) .
$$

The periodic orbit $y=\varphi(x)$ is transformed to the constant periodic orbit $z=0$. We denote by $\psi\left(x ; z_{0}\right)$ the solution of the latter differential equation with initial condition $\psi\left(0 ; z_{0}\right)=z_{0}$, and we obviously have that $\psi(x ; 0) \equiv 0$. We are interested in studying the behavior of the solutions of this equation in a neighborhood of the periodic orbit $z=0$, so we define the translation operator $\Psi\left(z_{0}\right)=\psi\left(1 ; z_{0}\right)-z_{0}$ and we study it near the point $z_{0}=0$. In case that $\Psi\left(z_{0}\right)$ is identically null, we deduce that the periodic orbit $z=0$ belongs to a continuum of periodic orbits. If $\Psi\left(z_{0}\right)$ is not identically null, by definition we have that the multiplicity of $z_{0}=0$ as a zero of $\Psi\left(z_{0}\right)$ corresponds to the multiplicity of $z=0$ as limit cycle of the differential equation. Through the change of variables $z=y-\varphi(x)$, this multiplicity coincides with the one of $y=\varphi(x)$ for the initial differential equation.

We expand $\psi\left(x ; z_{0}\right)$ in Taylor series in a neighborhood of $z_{0}=0$ :

$$
\psi\left(x ; z_{0}\right)=h_{1}(x) z_{0}+h_{2}(x) z_{0}^{2}+h_{3}(x) z_{0}^{3}+h_{4}(x) z_{0}^{4}+\mathcal{O}\left(z_{0}^{5}\right),
$$

where $h_{i}(x), i=1,2,3,4$, are differentiable functions with $h_{1}(0)=1$ and $h_{2}(0)=h_{3}(0)=h_{4}(0)=0$, because $\psi\left(0 ; z_{0}\right)=z_{0}$. We have that $\psi\left(x ; z_{0}\right)$ satisfies the equality

$$
\frac{\partial \psi\left(x ; z_{0}\right)}{\partial x}=\mathcal{F}\left(x, \psi\left(x ; z_{0}\right)+\varphi(x)\right)-\mathcal{F}(x, \varphi(x)),
$$


which we expand in Taylor series in a neighborhood of $z_{0}=0$. Since this identity needs to be satisfied for any value of $z_{0}$ in the considered neighborhood, we can equate the coefficients of the same powers of $z_{0}$. Thus, we obtain the following system of differential equations for the functions $h_{i}(x)$, $i=1,2,3,4$ :

$$
\begin{aligned}
& h_{1}^{\prime}(x)= \frac{\partial \mathcal{F}}{\partial y}(x, \varphi(x)) h_{1}(x) \\
& h_{2}^{\prime}(x)= \frac{\partial \mathcal{F}}{\partial y}(x, \varphi(x)) h_{2}(x)-\frac{1}{2} \frac{\partial^{2} \mathcal{F}}{\partial y^{2}}(x, \varphi(x)) h_{1}(x)^{2}, \\
& h_{3}^{\prime}(x)= \frac{\partial \mathcal{F}}{\partial y}(x, \varphi(x)) h_{3}(x)+\frac{\partial^{2} \mathcal{F}}{\partial y^{2}}(x, \varphi(x)) h_{1}(x) h_{2}(x)+ \\
&+\frac{1}{6} \frac{\partial^{3} \mathcal{F}}{\partial y^{3}}(x, \varphi(x)) h_{1}(x)^{3}, \\
& h_{4}^{\prime}(x)=\frac{\partial \mathcal{F}}{\partial y}(x, \varphi(x)) h_{4}(x)+\frac{\partial^{2} \mathcal{F}}{\partial y^{2}}(x, \varphi(x))\left(\frac{h_{2}(x)^{2}}{2}+h_{1}(x) h_{3}(x)\right)+ \\
&+\frac{1}{2} \frac{\partial^{3} \mathcal{F}}{\partial y^{3}}(x, \varphi(x)) h_{1}(x)^{2} h_{2}(x)+\frac{1}{24} \frac{\partial^{4} \mathcal{F}}{\partial y^{4}}(x, \varphi(x)) h_{1}(x)^{4} .
\end{aligned}
$$

The solution of this system of differential equations satisfies:

$$
\begin{aligned}
& h_{1}(x)=e^{\mathcal{D}_{1}(x)} \\
& h_{2}(x)=h_{1}(x) \mathcal{D}_{2}(x) / 2, \\
& h_{3}(x)=h_{1}(x) \mathcal{D}_{3}(x) / 6+h_{2}(x)^{2} / h_{1}(x), \\
& h_{4}(x)=h_{1}(x) \mathcal{D}_{4}(x) / 24-h_{2}(x)^{3} / h_{1}(x)^{2}+2 h_{2}(x) h_{3}(x) / h_{1}(x),
\end{aligned}
$$

where the functions $\mathcal{D}_{i}(x)$ are the ones that appear in the statement of the lemma. We observe that $h_{1}(x)$ is strictly positive for any value of $x$, so the former expressions are well-defined for any value of $x$. These expressions imply that $h_{1}(1)=1$ if, and only if, $\mathcal{D}_{1}(1)=0$. Moreover, fixed $k \in\{2,3,4\}$ we have that $h_{1}(1)=1$ and $h_{i}(1)=0$ for all $i$ such that $2 \leq i \leq k$ if, and only if, $\mathcal{D}_{i}(1)=0$ for all $i$ such that $1 \leq i \leq k$.

We note that the translation operator reads for

$$
\Psi\left(z_{0}\right)=\left(h_{1}(1)-1\right) z_{0}+h_{2}(1) z_{0}^{2}+h_{3}(1) z_{0}^{3}+h_{4}(1) z_{0}^{4}+\mathcal{O}\left(z_{0}^{5}\right),
$$

and therefore, $z_{0}=0$ is a limit cycle of multiplicity $m$ with $m \in\{1,2,3,4\}$ if, and only if, $\mathcal{D}_{i}(1)=0$ for all $i$ such that $1 \leq i<m$ and $\mathcal{D}_{m}(1) \neq 0$.

Finally, if $\mathcal{D}_{1}(1)=\mathcal{D}_{2}(1)=\mathcal{D}_{3}(1)=\mathcal{D}_{4}(1)=0$, we have that either $z=0$ is a limit cycle of multiplicity $\geq 5$ or it belongs to a continuum of periodic orbits. 
Lemma 5 allows us to prove the following result which characterizes the multiplicity of a periodic orbit in case of a linear or a Riccati differential equation. The proof of this result is also given in $[8,7]$ and we include it here for the sake of completeness. The limit cycles (with the corresponding multiplicities) of a linear or a Riccati differential equation are realizable by polynomial limit cycles as it is shown in the following proof.

Proof of Theorem 3. We first consider the linear differential equation

$$
\frac{d y}{d x}=A_{0}(x)+A_{1}(x) y .
$$

Let $y=\varphi(x)$ be a periodic solution of (3). By Lemma 5 we have that $y=\varphi(x)$ is a hyperbolic limit cycle if and only if $\alpha \neq 0$. On the other hand, $y=0$ is a hyperbolic limit cycle of linear differential equation $y^{\prime}=y$. So in order to complete the proof of statement (a) we claim that when $\alpha=0$ any periodic solution is contained in a continuum of periodic orbits. This completes the proof of statement (a).

Now we prove the claim. We integrate equation (3) and we have that any solution is given by

$$
y(x)=e^{\int_{0}^{x} A_{1}(\sigma) d \sigma}\left(y_{0}+\int_{0}^{x} e^{-\int_{0}^{s} A_{1}(\sigma) d \sigma} A_{0}(s) d s\right),
$$

where $y_{0}$ is a real constant. Since $y=\varphi(x)$ is a solution of $(3), \varphi(x)$ can be written as in the previous expression with $y_{0}=\varphi(0)$. Since $\alpha=0$ the condition $\varphi(0)=\varphi(1)$ implies that $\int_{0}^{1} e^{-\int_{0}^{s} A_{1}(\sigma) d \sigma} A_{0}(s) d s=0$. Therefore it follows that any solution $y(x)$ of $(3)$ satisfies $y(0)=y(1)$. We conclude that $y=\varphi(x)$ is contained in a continuum of periodic orbits. Hence the claim is proved.

We consider now the Riccati polynomial differential equation, i.e.

$$
\frac{d y}{d x}=A_{0}(x)+A_{1}(x) y+A_{2}(x) y^{2} .
$$

We assume that $y=\varphi(x)$ is a periodic solution of this differential equation and we are going to see that it cannot be a limit cycle of multiplicity $>2$. By Lemma 5 we have that if $\varphi(x)$ is a limit cycle of multiplicity $>2$ then $\beta=\mathcal{D}_{1}(1)=0$ and $\gamma=\mathcal{D}_{2}(1)=0$, where

$$
\begin{aligned}
& \mathcal{D}_{1}(x)=\int_{0}^{x} A_{1}(\sigma)+2 \varphi(\sigma) A_{2}(\sigma) d \sigma, \\
& \mathcal{D}_{2}(x)=\int_{0}^{x} e^{\mathcal{D}_{1}(s)} A_{2}(s) d s .
\end{aligned}
$$

On the other hand since we know $y=\varphi(x)$ a particular solution of the Riccati equation, we have that any other solution of this equation is of the form

$$
y(x)=\varphi(x)+\frac{e^{\mathcal{D}_{1}(x)}}{z_{0}-\int_{0}^{x} e^{\mathcal{D}_{1}(s)} A_{2}(s) d s},
$$


where $z_{0}$ is a real constant. Since $\beta=\gamma=0$, we deduce from (4) that any solution of the Riccati equation is periodic and we conclude that $y=\varphi(x)$ is a non isolated periodic orbit.

We also need to show that if there are two different periodic solutions, then they are either both hyperbolic limit cycles or they are contained in a continuum of periodic solutions. We assume that $y=\varphi(x)$ is a periodic orbit such that $\beta=0$ and that there exists another periodic orbit, that is, there exists a value of $z_{0}$ in (4) for which the solution is defined in $x \in[0,1]$ and $y(0)=y(1)$. Then for this solution $z_{0}-\int_{0}^{x} e^{\mathcal{D}_{1}(s)} A_{2}(s) d s \neq 0$ for any $x \in$ $[0,1]$. Moreover the condition $y(0)=y(1)$ implies that $\int_{0}^{1} e^{\mathcal{D}_{1}(s)} A_{2}(s) d s=$ 0 , where we have used that $\varphi(0)=\varphi(1)$. Therefore we conclude, by the reasoning given in the previous paragraph, that there is a continuum of periodic orbits in which $y=\varphi(x)$ is contained.

In order to finish with the proof of statement (b) we remark that $y^{\prime}=y(y-$ 1 ) is a Riccati equation with two polynomial hyperbolic limit cycles, and that $y^{\prime}=y^{2}$ is a Riccati equation with a polynomial double limit cycle.

The following proof uses ideas of Lins Neto in the work [7].

Proof of Theorem 4. The Abel differential equation $d y / d x=y^{2}+A_{3}(x) y^{3}$, where $A_{3}(x)$ is any polynomial in $\mathbb{R}[x]$, has $y=0$ as double limit cycle. This statement is proved by a straight application of Lemma 5 since $\mathcal{D}_{1}(x) \equiv 0$ and $\mathcal{D}_{2}(x)=2 x$ in this case.

We need to show that given any integer $m$ with $m \geq 3$, there exists a differential equation $d y / d x=A_{2}(x) y^{2}+A_{3}(x) y^{3}$ with $A_{2}(x)$ and $A_{3}(x)$ polynomials in $\mathbb{R}[x]$ such that $y=0$ is a limit cycle of multiplicity exactly $m$. We fix $A_{2}(x)=1-2 x$ and the differential equation reads for $d y / d x=$ $(1-2 x) y^{2}+A_{3}(x) y^{3}$. By Lemma 5 and for any polynomial $A_{3}(x)$ the periodic orbit $y=0$ is either a limit cycle of multiplicity $\geq 3$ or it belongs to a continuum of periodic orbits, because $\mathcal{D}_{1}(x) \equiv 0, \mathcal{D}_{2}(x)=x-x^{2}$ and, thus, $\mathcal{D}_{2}(1)=0$. In order to simplify notation, we define $A(x):=x-x^{2}$.

We observe that if $A_{3}(x) \equiv 0$, then

$$
\psi_{0}\left(x ; y_{0}\right)=\frac{y_{0}}{1-A(x) y_{0}}
$$

is the solution of the differential equation $d y / d x=(1-2 x) y^{2}$ with initial condition $\psi\left(0 ; y_{0}\right)=y_{0}$. Since $A(x)=x-x^{2}$ is such that $A(1)=0$, we have that $\psi\left(1 ; y_{0}\right)=y_{0}$ and, therefore, $y=0$ belongs to a continuum of periodic orbits. We consider a perturbation with a real parameter $\varepsilon$, of the previous equation with the continuum of periodic orbits and we will show that this perturbation can be chosen so that $y=0$ is a limit cycle of multiplicity $m$ with $m \geq 3$. 
We choose an integer $m$ with $m \geq 3$ and we write

$$
A_{3}(x)=\varepsilon \sum_{i=0}^{m-3} \tilde{b}_{i}(\varepsilon) A(x)^{i},
$$

which is a polynomial in $x$ of degree $2(m-3)$ and where the functions $\tilde{b}_{i}(\varepsilon)$, $i=0,1, \ldots, m-3$ are analytic in a neighborhood of $\varepsilon=0$, to be chosen in order to get the desired multiplicity. Again, in order to simplify notation we denote by $b(x, \varepsilon)=\sum_{i=0}^{m-3} \tilde{b}_{i}(\varepsilon) A(x)^{i}$ and by $b_{i}=\tilde{b}_{i}(0), i=0,1, \ldots, m-3$. Thus, we are considering the family of differential equations

$$
\frac{d y}{d x}=(1-2 x) y^{2}+\varepsilon b(x, \varepsilon) y^{3},
$$

where $\varepsilon \in \mathbb{R}$ with $0<|\varepsilon|<<1$. We are going to show that there always exists a polynomial $b(x, \varepsilon)$ and a punctured interval of $\varepsilon=0$ such that the periodic orbit $y=0$ is a limit cycle of multiplicity $m$ for equation (5).

We consider $y=\psi\left(x ; y_{0}, \varepsilon\right)$ the solution of equation (5) with initial condition $\psi\left(0 ; y_{0}, \varepsilon\right)=y_{0}$. By classical results on the dependence of parameters and initial conditions, we know that $\psi\left(x ; y_{0}, \varepsilon\right)$ is analytic in $\varepsilon$ and in $y_{0}$. Since $y=0$ is a periodic orbit of $(5)$, we have that $\psi(x ; 0, \varepsilon) \equiv 0$ for all $x, \varepsilon \in \mathbb{R}$. We fix an integer number $m$ with $m \geq 3$. We say that $y=0$ is $a$ limit cycle of (5) of multiplicity $m$ at first order in $\varepsilon$ if

$$
\psi\left(1 ; y_{0}, \varepsilon\right)-y_{0}=\varepsilon\left(c y_{0}^{m}+\mathcal{O}\left(y_{0}^{m+1}\right)\right)+\mathcal{O}\left(\varepsilon^{2}\right),
$$

with $c \in \mathbb{R}, c \neq 0$. On the other hand, we say that $y=0$ is a limit cycle of (5) of multiplicity $m$ (at any order of $\varepsilon$ ) if $\psi\left(1 ; y_{0}, \varepsilon\right)-y_{0}=f(\varepsilon) y_{0}^{m}+\mathcal{O}\left(y_{0}^{m+1}\right)$, where $f(\varepsilon)$ is an analytic function in a neighborhood of $\varepsilon=0$ with $f(0)=$ 0 and $f^{\prime}(0) \neq 0$. In these definitions, we have taken into account that $\psi\left(1 ; y_{0}, 0\right)=y_{0}$ because when $\varepsilon=0$, the periodic orbit $y=0$ belongs to a continuum of periodic orbits.

The proof is made in two steps. First we show that the values $b_{i}=\tilde{b}_{i}(0)$, $i=0,1, \ldots, m-3$, can be chosen so that $y=0$ is a limit cycle of $(5)$ of multiplicity $m$ at first order in $\varepsilon$. This part of the proof is the same as the one given in [7], now in a particular case. The second step consists in using the Implicit Function Theorem to show that we can find functions $\tilde{b}_{i}(\varepsilon)$, $i=0,1, \ldots, m-3$, such that $y=0$ is a limit cycle of multiplicity $m$ of (5), at any order of $\varepsilon$.

We expand the function $\psi\left(x ; y_{0}, \varepsilon\right)$ in Taylor series in a neighborhood of $\varepsilon=0: \quad \psi\left(x ; y_{0}, \varepsilon\right)=\psi_{0}\left(x ; y_{0}\right)+\varepsilon \psi_{1}\left(x ; y_{0}\right)+\mathcal{O}\left(\varepsilon^{2}\right)$. The condition $\psi\left(0 ; y_{0}, \varepsilon\right)=y_{0}$ implies that $\psi_{0}\left(0 ; y_{0}\right)=y_{0}$ and $\psi_{1}\left(0 ; y_{0}\right)=0$. We expand the equality

$$
\frac{\partial \psi\left(x ; y_{0}, \varepsilon\right)}{\partial x}=(1-2 x) \psi\left(x ; y_{0}, \varepsilon\right)^{2}+\varepsilon b(x, \varepsilon) \psi\left(x ; y_{0}, \varepsilon\right)^{3},
$$

in power series of $\varepsilon$ and we equate the coefficients of the powers $\varepsilon^{0}$ and $\varepsilon^{1}$. We deduce that $\psi_{0}\left(x ; y_{0}\right)=y_{0} /\left(1-A(x) y_{0}\right)$, as we have seen before, and 
that

$$
\psi_{1}\left(x ; y_{0}\right)=\psi_{0}\left(x ; y_{0}\right)^{2} \int_{0}^{x} b(s, 0) \psi_{0}\left(s ; y_{0}\right) d s .
$$

We remark that $y=0$ is a limit cycle of (5) of multiplicity $m$ at first order in $\varepsilon$ if $\psi_{1}\left(1 ; y_{0}\right)=c y_{0}^{m}+\mathcal{O}\left(y_{0}^{m+1}\right)$ with $c \in \mathbb{R}, c \neq 0$. We evaluate $\psi_{1}\left(x ; y_{0}\right)$ in $x=1$ and we recall that $\psi_{0}\left(1 ; y_{0}\right)=y_{0}$. We have that

$$
\psi_{1}\left(1 ; y_{0}\right)=y_{0}^{3} \int_{0}^{1} \frac{b(s, 0)}{1-A(s) y_{0}} d s .
$$

We define the values $\mathcal{I}_{k}=\int_{0}^{1} A(s)^{k} d s$, for any integer $k$ with $k \geq 0$. Since $b(s, 0)=b_{0}+b_{1} A(s)+b_{2} A(s)^{2}+\ldots+b_{m-3} A(s)^{m-3}$, we can write

$$
\begin{aligned}
\psi_{1}\left(1 ; y_{0}\right) & =y_{0}^{3}\left[\int_{0}^{1}\left(\sum_{i=0}^{m-3} b_{i} A(s)^{i}\right)\left(\sum_{j=0}^{m-3} A(s)^{j} y_{0}^{j}+\mathcal{O}\left(y_{0}\right)^{m-2}\right) d s\right] \\
& =y_{0}^{3}\left[\sum_{j=0}^{m-3}\left(\int_{0}^{1}\left(\sum_{i=0}^{m-3} b_{i} A(s)^{i+j}\right) d s\right) y_{0}^{j}+\mathcal{O}\left(y_{0}^{m-2}\right)\right] \\
& =y_{0}^{3}\left[\sum_{j=0}^{m-3}\left(\sum_{i=0}^{m-3} b_{i} \mathcal{I}_{i+j}\right) y_{0}^{j}+\mathcal{O}\left(y_{0}^{m-2}\right)\right] .
\end{aligned}
$$

We consider the system of $m-2$ linear equations with the $(m-2) \times(m-2)$ matrix $\mathcal{M}=\left(m_{i j}\right)=\left(\mathcal{I}_{i+j}\right)$, for $0 \leq i, j \leq m-3$, the vector of $m-2$ unknowns $\left(b_{0}, b_{1}, b_{2}, \ldots, b_{m-3}\right)$ and independent vector $\left(0,0, \ldots{ }^{(m-3)}, 0,1\right)$. If we show that the determinant of $\mathcal{M}$ is different from zero, we will have that this system always has a unique solution and, thus, we can always find values of $b_{0}, b_{1}, \ldots, b_{m-3}$ for which $\psi_{1}\left(1 ; y_{0}\right)=y_{0}^{m}+\mathcal{O}\left(y_{0}^{m+1}\right)$. We consider the vector space $\mathcal{S}$ of real polynomials generated linear combinations of $A(x)^{j}$, with $j=0,1, \ldots, m-3$, and where we recall that $A(x)=x-x^{2}$. Then

$$
\mathcal{S}=\left\{\sum_{j=0}^{m-3} c_{j} A(x)^{j}: c_{j} \in \mathbb{R}\right\},
$$

is a real vector space of dimension $m-2$. Given $f(x), g(x) \in \mathcal{S}$, we can define the inner product of $f(x)$ and $g(x)$ by $\langle f, g\rangle=\int_{0}^{1} f(x) g(x) d x$. It is clear that $A(x)^{j}$, with $j=0,1, \ldots, m-3$ forms a basis of $\mathcal{S}$ and that $\mathcal{M}$ is the symmetric matrix of the metrics corresponding to this inner product. Therefore $\operatorname{det}(\mathcal{M}) \neq 0$.

We have proved that we can always find a polynomial $b(x, 0)$ such that the differential equation (5) has $y=0$ as limit cycle of multiplicity $m$, at first order in $\varepsilon$. We will use $b(x, \varepsilon)$ as a function in $\varepsilon$ in order to show the result at any order of $\varepsilon$. We consider again the solution $y=\psi\left(x ; y_{0}, \varepsilon\right)$ of 
equation (5), which is analytic in a neighborhood of the initial condition $y_{0}=0$. We have that $\psi(x ; 0, \varepsilon) \equiv 0$. We expand the function $\psi\left(x ; y_{0}, \varepsilon\right)$ in Taylor series in a neighborhood of $y_{0}=0$ :

$$
\psi\left(x ; y_{0}, \varepsilon\right)=\tilde{\varphi}_{1}(x ; \varepsilon, b) y_{0}+\tilde{\varphi}_{2}(x ; \varepsilon, b) y_{0}^{2}+\mathcal{O}\left(y_{0}^{3}\right),
$$

where we remark the dependence on the parameters $b$. The condition $\psi\left(0 ; y_{0}, \varepsilon\right)=y_{0}$ implies that $\tilde{\varphi}_{1}(0 ; \varepsilon, b)=1$ and $\tilde{\varphi}_{2}(0 ; \varepsilon, b)=0$. Equating the coefficients of $y_{0}$ and $y_{0}^{2}$ in the equality

$$
\frac{\partial \psi\left(x ; y_{0}, \varepsilon\right)}{\partial x}=(1-2 x) \psi\left(x ; y_{0}, \varepsilon\right)^{2}+\varepsilon b(x, \varepsilon) \psi\left(x ; y_{0}, \varepsilon\right)^{3},
$$

we deduce that $\tilde{\varphi}_{1}(x ; \varepsilon, b) \equiv 1$ and $\tilde{\varphi}_{2}(x ; \varepsilon, b)=A(x)=x-x^{2}$. On the other hand, $\psi\left(1 ; y_{0}, 0\right)=y_{0}$, so we can write

$$
\psi\left(1 ; y_{0}, \varepsilon\right)=y_{0}+\varepsilon \sum_{i=3}^{m} \varphi_{i}(1 ; \varepsilon, b) y_{0}^{i}+\mathcal{O}\left(y_{0}^{m+1}\right),
$$

where we recall that $A(1)=0$ and that $\varphi_{i}(0 ; \varepsilon, b)=0$ for $i \geq 3$. In order to have $y=0$ as a limit cycle of multiplicity $m$, we are going to show that we can solve the following system of $m-2$ equations $\varphi_{i}(1 ; \varepsilon, b)=0$ for $i=3,4, \ldots, m-1$ and $\varphi_{m}(1 ; \varepsilon, b)=1$. We consider the function $\Phi(\varepsilon, b)$ : $\mathbb{R} \times \mathbb{R}^{m-2} \rightarrow \mathbb{R}$ defined by $\Phi(\varepsilon, b)=\left(\varphi_{3}(1 ; \varepsilon, b), \varphi_{4}(1 ; \varepsilon, b), \ldots, \varphi_{m}(1 ; \varepsilon, b)\right)$, where we treat $b$ as the $m-2$ variables given by the coefficients $\tilde{b}_{i}$, with $i=0,1,2, \ldots, m-3$.

As we have defined $\psi\left(x ; y_{0}, \varepsilon\right)=\psi_{0}\left(x ; y_{0}\right)+\varepsilon \psi_{1}\left(x ; y_{0}\right)+\mathcal{O}\left(\varepsilon^{2}\right)$, which implies that $\sum_{i=3}^{m} \varphi_{i}(1 ; \varepsilon, b) y_{0}^{i}+\mathcal{O}\left(y_{0}^{m+1}\right)=\psi_{1}\left(1 ; y_{0}\right)+\mathcal{O}(\varepsilon)$. In the previous paragraph we have shown that $\varphi_{i}(1 ; 0, b)=\sum_{j=0}^{m-3} \mathcal{I}_{i+j} b_{j}+\mathcal{O}(\varepsilon)$. We consider the values $b_{*}=\left(b_{0}, b_{1}, \ldots, b_{m-3}\right)$ described in the previous paragraph, that is, the solution of the linear system of equations with the matrix $\mathcal{M}$ and independent vector $\left(0,0, \ldots{ }^{(m-3)}, 0,1\right)$. We deduce that $\Phi\left(0, b_{*}\right)=\left(0,0, \ldots{ }^{(m-3)}, 0,1\right)$ and that the Jacobian matrix $D_{b} \Phi(\varepsilon, b)$ corresponding to the derivation only with respect to the variables $b$ satisfies $D_{b} \Phi\left(0, b_{*}\right)=\mathcal{M}$. Thus its determinant is different from zero. By the Implicit Function Theorem, we can ensure the existence of $m-2$ functions $\tilde{b}_{0}(\varepsilon)$, $\tilde{b}_{1}(\varepsilon), \ldots \tilde{b}_{m-3}(\varepsilon)$ analytic in a neighborhood of $\varepsilon=0$, such that $\tilde{b}_{i}(0)=b_{i}$ for $i=0,1, \ldots, m-3$, and which satisfy

$$
\Phi\left(\varepsilon, \tilde{b}_{0}(\varepsilon), \tilde{b}_{1}(\varepsilon), \ldots, \tilde{b}_{m-3}(\varepsilon)\right) \equiv\left(0,0, \ldots{ }^{(m-3)}, 0,1\right),
$$

for any $\varepsilon$ in a neighborhood of $\varepsilon=0$.

\section{ACKNOWLEDGEMENTS}

The first and second authors are partially supported by a MCYT/FEDER grant number MTM2005-06098-C02-02. The first author is also partially supported by a CIRIT grant number 2005SGR 00550, and by DIUE of Government of Catalonia "Distinció de la Generalitat de Catalunya per a la 
promoció de la recerca universitària". The third author is partially supported by a MCYT/FEDER grant number MTM2005-06098-C02-01 and by a CIRIT grant number 2005SGR 00550.

\section{REFERENCES}

[1] D. Behloul And S. S. Cheng, Computation of all polynomial solutions of a class of nonlinear differential equations, Computing 77 (2006), 163-177.

[2] M. Briskin, J.P. FrançOise And Y. Yomdin, Center conditions, compositions of polynomials and moments on algebraic curves, Ergodic Theory \& Dynam. Systems 19 (1999), 1201-1220.

[3] M. Briskin, J.P. Françoise and Y. Yomdin, Center conditions. II. Parametric and model center problems, Israel J. Math. 118 (2000), 61-82.

[4] M. Briskin, J.P. Françoise And Y. Yomdin, Center conditions. III. Parametric and model center problems, Israel J. Math. 118 (2000), 83-108.

[5] J.G. Campbell and M. Golomb, On the polynomial solutions of a Riccati equation, The Amer. Math. Monthly 61 (1954), 402-404.

[6] A. Gasull And J. Llibre, Limit cycles for a class of Abel equations, SIAM J. Math. Anal. 21 (1990), 1235-1244.

[7] A. Lins Neto, On the number of solutions of the equation $d x / d t=\sum_{j=0}^{n} a_{j}(t) x^{j}$, $0 \leq t \leq 1$, for which $x(0)=x(1)$, Invent. Math. 59 (1980), 67-76.

[8] N.G. LlOYD, The number of periodic solutions of the equation $\dot{z}=z^{N}+p_{1}(t) z^{N-1}+$ $\ldots+p_{N}(t)$, Proc. London Math. Soc. 27 (1973), 667-700.

[9] N.G. LlOYD, A note on the number of limit cycles in certain two-dimensional systems, J. London Math. Soc. 20 (1979), 277-286.

[10] E.D. RAINville, Necessary conditions for polynomial solutions of certain Riccati equations, The Amer. Math. Monthly 43 (1936), 473-476.

1 Departament de Matemàtica, Universitat de Lleida, Avda. Jaume II, 69; 25001 Lleida, Catalonia, Spain

E-mail address: gine@matematica.udl.cat, mtgrau@matematica.udl.cat

2 Departament de Matemàtiques, Universitat Autònoma de Barcelona, 08193 Bellaterra, Barcelona, Catalonia, Spain

E-mail address: jllibre@mat.uab.cat 